\title{
NILPOTENT GROUPS WITHOUT EXACTLY POLYNOMIAL DEHN FUNCTION
}

\author{
STEFAN WENGER
}

\begin{abstract}
Aвstract. We prove super-quadratic lower bounds for the growth of the filling area function of a certain class of Carnot groups. This class contains groups for which it is known that their Dehn function grows no faster than $n^{2} \log n$. We therefore obtain the existence of (finitely generated) nilpotent groups whose Dehn functions do not have exactly polynomial growth and we thus answer a well-known question about the possible growth rate of Dehn functions of nilpotent groups.
\end{abstract}

\section{INTRODUCTION}

Dehn functions have played an important role in geometric group theory. They measure the complexity of the word problem in a given finitely presented group and provide a quasiisometry invariant of the group. A class of groups for which the Dehn function has been well-studied is that of nilpotent groups. Many results on upper bounds for the growth of the Dehn function are known, while fewer techniques have been found for obtaining lower bounds. Here are some known results: if $\Gamma$ is a finitely generated nilpotent group of step $c$ then its Dehn function $\delta_{\Gamma}(n)$ grows no faster than $n^{c+1}$, in short $\delta_{\Gamma}(n) \leq n^{c+1}$, [12, 20, 9]. If $\Gamma$ is a free nilpotent group of step $c$ then $\delta_{\Gamma}(n) \sim n^{c+1},[5,21]$. This includes the particular case of the first Heisenberg group, which was known before, see [8]. For the definition of $\delta_{\Gamma}(n)$ and the meaning of $\leq$ and $\sim$ see Section 2 The higher Heisenberg groups have quadratic Dehn functions, [12, 1, 16]. Ol'shanskii-Sapir used the arguments in their paper [16] to prove that the Dehn function of the central product of $m \geq 2$ copies of a nilpotent group of step 2 grows no faster than $n^{2} \log n$. This has also recently been proved in [27] with different methods.

The following question about the possible growth rate of the Dehn function of nilpotent groups has been raised by many authors, see e.g. [21, 4, 6], but has remained open so far.

Question 1.1. Does the Dehn function of every finitely generated nilpotent group $\Gamma$ grow exactly polynomially, that is,

$$
\delta_{\Gamma}(n) \sim n^{\alpha} \quad \text { for some (integer) } \alpha ?
$$

The primary purpose of this article is to prove super-quadratic lower bounds for the growth of the Dehn function of certain classes of nilpotent groups and to combine these with the results from [16, 27] to give a negative answer to the above question. We establish:

Theorem 1.2. There exist finitely generated nilpotent groups $\Gamma$ of step 2 whose Dehn function $\delta_{\Gamma}(n)$ satisfies

$$
n^{2} \varrho(n) \leq \delta_{\Gamma}(n) \leq n^{2} \log n
$$

for some function $\varrho$ with $\varrho(n) \rightarrow \infty$ as $n \rightarrow \infty$.

Date: April 16, 2010.

Partially supported by NSF grant DMS 0956374. 
We can in fact produce a whole family of groups satisfying (1). They all arise as lattices of (central powers) of Carnot groups of step 2, and the upper bound comes as a consequence of [16, 27]. Recall that a simply connected nilpotent Lie group is called Carnot group if its Lie algebra admits a grading, the first layer of which generates the whole Lie algebra, see Section 2.3 for definitions. In Theorem 4.3 we will show that under suitable conditions on the Lie algebra of a Carnot group $G$ of step 2 the $m$-th central power of $G$ has filling area function which grows strictly faster than quadratically. Recall that the filling area function $\mathrm{FA}(r)$ of a simply connected Riemannian manifold $X$ is the smallest function such that every closed curve in $X$ of length at most $r$ bounds a singular Lipschitz chain of area at most FA $(r)$. As is well-known, FA $(r)$ bounds from below the Dehn function of any finitely generated group acting properly discontinuously and cocompactly by isometries on $X$.

In preparation of the proof of the super-quadratic lower bounds and of Theorem 1.2, we will prove, in Section 3 , the following theorem which should be of independent interest.

Theorem 1.3. Let $X$ be a simply connected Riemannian manifold with quadratic filling area function, $\mathrm{FA}(r) \leq r^{2}$. Then every asymptotic cone $X_{\omega}$ of $X$ admits a quadratic isoperimetric inequality for integral 1-currents in $X_{\omega}$. In particular, there exists $C>0$ such that every closed Lipschitz curve $c$ in $X_{\omega}$ bounds an integral 2-current in $X_{\omega}$ of mass ("area") at most $C$ length $(c)^{2}$.

The above theorem holds more generally for complete metric length spaces $X$, see Theorem 3.1 The theory of integral currents in metric spaces was developed by AmbrosioKirchheim in [3]. We refer to Section 2 for definitions. Integral 2-currents should be thought of as generalized singular Lipschitz chains and the mass is proportional to the Hausdorff area. A consequence of Theorem 1.3 is the following:

Corollary 1.4. Let $\Gamma$ be a finitely presented group with quadratic Dehn function. Then every asymptotic cone of $\Gamma$ admits a quadratic isoperimetric inequality for integral 1currents.

It is known that finitely presented groups with quadratic Dehn function have simply connected asymptotic cones, [19]. Many groups, however, have simply connected asymptotic cones but do not have quadratic Dehn function. Corollary 1.4 does not yield simple connectedness but instead provides strong metric information. This can be used to prove that certain groups cannot admit quadratic Dehn function, see below.

We now briefly describe how Theorem 1.3 will be used to obtain super-quadratic growth estimates for filling area functions. Let $H$ be a Carnot group and let $d_{0}$ be the distance induced by a left-invariant Riemannian metric on $H$. Denote by $d_{c}$ the Carnot-Carathéodory distance on $H$ associated with $d_{0}$, see Section 2.3 By [17], the metric space $\left(H, d_{c}\right)$ is the unique asymptotic cone of $\left(H, d_{0}\right)$. In order to prove that the filling area function of $\left(H, d_{0}\right)$ grows strictly faster than quadratically for a given $\left(H, d_{0}\right)$ it thus suffices, by Theorem 1.3 , to prove that the metric space $\left(H, d_{c}\right)$ cannot admit a quadratic isoperimetric inequality for integral 1-currents. In Sections 4 and 5 we will show that under suitable conditions on the Lie algebra of $H$, the metric space $\left(H, d_{c}\right)$ has non-trivial first homology group for integral currents, and in particular $\left(H, d_{c}\right)$ does not admit a quadratic isoperimetric inequality for integral 1-currents. The conditions exhibited in Section 4 will be used to establish the lower bounds needed to prove Theorem 1.2 , they can roughly be described as follows. Let $G$ be a Carnot group of step 2 with grading $\mathfrak{g}=V_{1} \oplus V_{2}$ of its Lie algebra. Given a proper subspace $U \subset V_{2}$, one obtains a new Lie algebra $\mathfrak{g}_{U}=V_{1} \oplus\left(V_{2} / U\right)$ and hence a Carnot group $G_{U}$ with Lie algebra $\mathfrak{g}_{U}$. Note that every simply connected nilpotent Lie group of step 2 arises this way with $\mathfrak{g}=V_{1} \oplus V_{2}$ a free nilpotent Lie algebra of step 2 and $U \subset V_{2}$ a 
suitable subspace. In the proof of Theorem 4.3 we will show that under a suitable condition on $U$, which we term $m$-inaccessibility, the $m$-th central power $H:=G_{U} \times_{Z} \cdots \times_{Z} G_{U}$, endowed with a Carnot-Carathéodory distance, has non-trivial first Lipschitz homology. In view of the above, this is enough to conclude that the filling area function of $H$ grows super-quadratically when $H$ is endowed with a left-invariant Riemannian metric. The $m$ inaccessibility condition is given in Definition 4.1, it is not difficult to provide examples of $m$-inaccessible subspaces $U$, see Example 4.2 and the remark following it. For the proofs of Theorems 4.3 and 1.2, we only need the special case of Theorem 1.3 when $X$ is a Carnot group endowed with a left-invariant Riemannian metric. In this case, the proof can be simplified and we can in fact prove an analog for cycles of any dimension, see Proposition 3.6. We believe that, nevertheless, the generality of Theorem 1.3 should be of interest, especially since not much is known about groups with quadratic Dehn function.

The paper is structured as follows: Section 2 contains definitions of and facts about Dehn functions, filling area functions, asymptotic cones, and Carnot groups. We will also recall all definitions from Ambrosio-Kirchheim's theory of integral currens [3] which we will need later. In Section 3 we will use the theory of currents to prove Theorem 1.3 and the more general Theorem 3.1 In Section 4, Theorem 3.1 will be used to prove the superquadratic lower bounds in Theorem 4.3 and to establish Theorem 1.2 Finally, in Section 5 we use Theorem 3.1 to exhibit super-quadratic lower bounds for another class of Carnot groups, not necessarily of step 2, see Theorem 5.1. The results obtained in Section 5 are not needed for the proof of Theorem 1.2 .

Acknowledgment: I am indebted to Robert Young for inspiring discussions and for bringing Question 1.1 to my attention. Parts of the research underlying this paper was carried out during a research visit to the Université Catholique de Louvain, Belgium. I wish to thank the Mathematics Department and Thierry de Pauw for the hospitality.

\section{Preliminaries}

The purpose of this section is to collect some definitions and facts which will be used in the sequel.

2.1. Dehn and filling area functions. Let $\Gamma=\langle S \mid R\rangle$ be a finitely presented group. The Dehn function $\delta_{\Gamma}(n)$ of $\Gamma$ with respect to the given presentation is defined by

$$
\delta_{\Gamma}(n):=\max _{w=\Gamma 1,|w| \leq n} \min \left\{k: w=\prod_{i=1}^{k} g_{i} r_{i}^{ \pm 1} g_{i}^{-1}, r_{i} \in R, g_{i} \text { word in } S\right\} .
$$

Here, $w$ is a word in the alphabet $S$ and $|w|$ denotes the word length of $w$. The equality $w=\prod_{i=1}^{k} g_{i} r_{i}^{ \pm 1} g_{i}^{-1}$ is in the free group generated by $S$. For functions $f, g: \mathbb{N} \rightarrow \mathbb{N}$ or $f, g:[0, \infty] \rightarrow(0, \infty)$ one writes $f \leq g$ if there exists $C$ such that

$$
f(r) \leq \mathrm{Cg}(\mathrm{Cr}+\mathrm{C})+\mathrm{Cr}+\mathrm{C}
$$

for all $r>0$. One furthermore writes $f \sim g$ if $f \leq g$ and $g \leq f$. If $\Gamma$ acts properly discontinuously and cocompactly by isometries on a simply connected Riemannian manifold $X$ then

$$
\delta_{\Gamma}(n) \sim \mathrm{FA}_{0}(n),
$$

where the Dehn function $\mathrm{FA}_{0}(r)$ on $X$ is defined by

$$
\mathrm{FA}_{0}(r):=\sup \left\{\text { Fillarea }_{0}(c): c \text { closed Lipschitz curve in } X \text { of length }(c) \leq r\right\},
$$


and Fillarea $0(c)$ of $c: S^{1} \rightarrow X$ is given by

$$
\text { Fillarea }_{0}(c):=\inf \left\{\operatorname{Area}(\varphi): \varphi: D^{2} \rightarrow X \text { Lipschitz, }\left.\varphi\right|_{S^{1}}=c\right\} .
$$

Here, $\operatorname{Area}(\varphi)$ is the integral over the disc $D^{2}$ of the jacobian of $\varphi$. The filling area function $\mathrm{FA}(r)$ of $X$ is defined by

$$
\mathrm{FA}(r):=\sup \{\text { Fillarea }(c): c \text { closed Lipschitz curve in } X \text { of length }(c) \leq r\},
$$

where

Fillarea $(c):=\inf \{\operatorname{Area}(z): z$ is a singular Lipschitz 2-chain in $X$ with boundary $c\}$.

Recall that a singular Lipschitz 2-chain is a formal finite sum $z=\sum m_{i} \varphi_{i}$ with $m_{i} \in \mathbb{Z}$ and $\varphi_{i}: \Delta^{2} \rightarrow X$ Lipschitz and its area is

$$
\operatorname{Area}(z):=\sum\left|m_{i}\right| \operatorname{Area}\left(\varphi_{i}\right)
$$

see [11]. It is clear that

$$
\mathrm{FA}(r) \leq \mathrm{FA}_{0}(r)
$$

for all $r \geq 0$.

2.2. Ultralimits and asymptotic cones of metric spaces. Recall that a non-principal ultrafilter on $\mathbb{N}$ is a finitely additive probability measure $\omega$ on $\mathbb{N}$ (together with the $\sigma$-algebra of all subsets) such that $\omega$ takes values in $\{0,1\}$ only and $\omega(A)=0$ whenever $A \subset \mathbb{N}$ is finite. The existence of non-principal ultrafilters on $\mathbb{N}$ follows from Zorn's lemma. It is not difficult to prove the following fact. If $(Y, \tau)$ is a compact topological Hausdorff space then for every sequence $\left(y_{n}\right)_{n \in \mathbb{N}} \subset Y$ there exists a unique point $y \in Y$ such that

$$
\omega\left(\left\{n \in \mathbb{N}: y_{n} \in U\right\}\right)=1
$$

for every $U \in \tau$ containing $y$. We will denote this point by $\lim _{\omega} y_{n}$.

Let now $\left(X_{n}, d_{n}, p_{n}\right)$ be pointed metric spaces, $n \geq 1$, and fix a non-principal ultrafilter $\omega$ on $\mathbb{N}$. A sequence $\left(x_{n}\right)$, where $x_{n} \in X_{n}$ for each $n$, will be called bounded if

$$
\sup _{n} d_{n}\left(p_{n}, x_{n}\right)<\infty \text {. }
$$

Define an equivalence relation on bounded sequences by

$$
\left(x_{n}\right) \sim\left(x_{n}^{\prime}\right) \text { if and only if } \lim _{\omega} d_{n}\left(x_{n}, x_{n}^{\prime}\right)=0 .
$$

Let $X_{\omega}$ be the set of equivalence classes of bounded sequences, $d_{\omega}$ the metric on $X_{\omega}$ given by

$$
d_{\omega}\left(\left[\left(x_{n}\right)\right],\left[\left(x_{n}^{\prime}\right)\right]\right):=\lim _{\omega} d_{n}\left(x_{n}, x_{n}^{\prime}\right),
$$

and $p_{\omega}:=\left[\left(p_{n}\right)\right]$. Then the pointed metric space $\left(X_{\omega}, d_{\omega}, p_{\omega}\right)$ is called the ultralimit of the sequence $\left(X_{n}, d_{n}, p_{n}\right)$ with respect to $\omega$. If $(X, d)$ is a metric space, $\left(p_{n}\right) \subset X$ a sequence of basepoints, and $r_{n}>0$ satisfies $r_{n} \rightarrow \infty$, then the ultralimit of the sequence $\left(X, r_{n}^{-1} d, p_{n}\right)$ with respect to $\omega$ is called the asymptotic cone of $\left(X,\left(r_{n}\right),\left(p_{n}\right)\right)$ with respect to $\omega$.

It is not difficult to show that ultralimits are always complete. Furthermore, if every $\left(X_{n}, d_{n}\right)$ is a length space, then the ultralimits are all length spaces as well. 
2.3. Carnot groups and their asymptotic cones. Recall that a connected and simply connected nilpotent Lie group $G$ of step $k$ is called Carnot group if its Lie algebra $g$ admits a grading

$$
\mathfrak{g}=V_{1} \oplus \cdots \oplus V_{k}
$$

such that $\left[V_{1}, V_{i}\right]=V_{i+1}$ for all $i=1, \ldots, k-1$ and $\left[V_{1}, V_{k}\right]=0$, where $\left[V_{1}, V_{i}\right]$ is the smallest subspace spanned by the elements $\left[v, v^{\prime}\right]$ with $v \in V_{1}$ and $v^{\prime} \in V_{i}$. In other words, a Carnot group is a homogeneous nilpotent Lie group such that the first layer $V_{1}$ of its Lie algebra generates the entire Lie algebra. Clearly, every connected and simply connected nilpotent Lie group of step 2 is a Carnot group. Let now $G$ be a Carnot group of step $k$ and let

$$
\mathfrak{g}=V_{1} \oplus \cdots \oplus V_{k}
$$

be a grading of its Lie algebra $\mathfrak{g}$. Note that the exponential map exp $: \mathfrak{g} \rightarrow G$ is a diffeomorphism. $G$ comes with a family of dilation homomorphisms $\delta_{r}: G \rightarrow G, r \geq 0$, which, on the level of Lie algebras, take the form

$$
\delta_{r}(v)=\sum_{i=1}^{k} r^{i} v_{i}
$$

for $v=v_{1}+\cdots+v_{k}$ with $v_{i} \in V_{i}$.

Let $g_{0}$ be a left-invariant Riemannian metric on $G$ and denote by $d_{0}$ the distance on $G$ coming from $g_{0}$. A new distance, called Carnot-Carathéodory distance, can be associated with $g_{0}$ as follows. Define the horizontal subbundle $T H$ of $T G$ by left-translating $V_{1}$. A curve $c:[0,1] \rightarrow G$, absolutely continuous (ac for short) with respect to $d_{0}$, is called horizontal if $\dot{c}(t) \in T_{c(t)} H$ for almost every $t \in[0,1]$. The Carnot-Carathéodory distance $d_{c}$ on $G$ associated with $g_{0}$ is then defined by

$$
d_{c}(x, y)=\inf \left\{\text { length }_{g_{0}}(c): c \text { horizontal ac curve joining } x \text { to } y\right\}
$$

where length $g_{0}(c)$ denotes the length of $c$ with respect to $g_{0}$. It can be shown that $d_{c}$ defines a metric, ie. that $d_{c}(x, y)$ is always finite. Important properties of the Carnot-Carathéodory distance are that it is left invariant and 1-homogeneous with respect to the dilations, i.e., $d_{c}\left(\delta_{r}(x), \delta_{r}(y)\right)=r d_{c}(x, y)$ for all $x, y \in G$ and all $r \geq 0$. We also obviously have the relationship

$$
d_{0} \leq d_{c}
$$

and it is well-known that $d_{0}$ and $d_{c}$ are not bi-Lipschitz equivalent unless $G$ is Euclidean. Note however, that the topologies induced by $d_{0}$ and $d_{c}$ are the same.

The following theorem, which is a special case of a more general result due to Pansu [17], gives a link between left-invariant Riemannian metrics and Carnot-Carathéodory metrics on Carnot groups.

Theorem 2.1. Let $G$ be a Carnot group and let $d_{0}$ be the distance associated with a left-invariant Riemannian metric $g_{0}$ on $G$. Then the pointed spaces $\left(G, \frac{1}{r} d_{0}, e\right)$ converge in the pointed Gromov-Hausdorff sense to $\left(G, d_{c}, e\right)$ as $r \rightarrow \infty$, where $d_{c}$ is the CarnotCarathéodory distance on $G$ associated with $g_{0}$. Here, e denotes the identity element of $G$.

In particular, it follows that $\left(G, d_{0}\right)$ has a unique asymptotic cone, which moreover is isometric to $\left(G, d_{c}\right)$. This will be used in the proofs of Theorem 4.3 and Theorem 5.1 
2.4. Integral currents in metric spaces. The theory of integral currents in metric spaces was developed by Ambrosio and Kirchheim in [3] and provides a suitable notion of surfaces and area/volume in the setting of metric spaces. In the following we recall the definitions that are needed for our purposes.

Let $(Y, d)$ be a complete metric space and $m \geq 0$ and let $\mathcal{D}^{m}(Y)$ be the set of $(m+1)$-tuples $\left(f, \pi_{1}, \ldots, \pi_{m}\right)$ of Lipschitz functions on $Y$ with $f$ bounded. The Lipschitz constant of a Lipschitz function $f$ on $Y$ will be denoted by $\operatorname{Lip}(f)$.

Definition 2.2. An m-dimensional metric current $T$ on $Y$ is a multi-linear functional on $\mathcal{D}^{m}(Y)$ satisfying the following properties:

(i) If $\pi_{i}^{j}$ converges point-wise to $\pi_{i}$ as $j \rightarrow \infty$ and if $\sup _{i, j} \operatorname{Lip}\left(\pi_{i}^{j}\right)<\infty$ then

$$
T\left(f, \pi_{1}^{j}, \ldots, \pi_{m}^{j}\right) \longrightarrow T\left(f, \pi_{1}, \ldots, \pi_{m}\right) .
$$

(ii) If $\{y \in Y: f(y) \neq 0\}$ is contained in the union $\bigcup_{i=1}^{m} B_{i}$ of Borel sets $B_{i}$ and if $\pi_{i}$ is constant on $B_{i}$ then

$$
T\left(f, \pi_{1}, \ldots, \pi_{m}\right)=0 .
$$

(iii) There exists a finite Borel measure $\mu$ on $Y$ such that

$$
\left|T\left(f, \pi_{1}, \ldots, \pi_{m}\right)\right| \leq \prod_{i=1}^{m} \operatorname{Lip}\left(\pi_{i}\right) \int_{Y}|f| d \mu
$$

for all $\left(f, \pi_{1}, \ldots, \pi_{m}\right) \in \mathcal{D}^{m}(Y)$.

The space of $m$-dimensional metric currents on $Y$ is denoted by $\mathbf{M}_{m}(Y)$ and the minimal Borel measure $\mu$ satisfying (2) is called mass of $T$ and written as $\|T\|$. We also call mass of $T$ the number $\|T\|(Y)$ which we denote by $\mathbf{M}(T)$. The support of $T$ is, by definition, the closed set spt $T$ of points $y \in Y$ such that $\|T\|(B(y, r))>0$ for all $r>0$.

Every function $\theta \in L^{1}(K, \mathbb{R})$ with $K \subset \mathbb{R}^{m}$ Borel measurable induces an element of $\mathbf{M}_{m}\left(\mathbb{R}^{m}\right)$ by

$$
\llbracket \theta \rrbracket\left(f, \pi_{1}, \ldots, \pi_{m}\right):=\int_{K} \theta f \operatorname{det}\left(\frac{\partial \pi_{i}}{\partial x_{j}}\right) d \mathcal{L}^{m}
$$

for all $\left(f, \pi_{1}, \ldots, \pi_{m}\right) \in \mathcal{D}^{m}\left(\mathbb{R}^{m}\right)$.

The restriction of $T \in \mathbf{M}_{m}(Y)$ to a Borel set $A \subset Y$ is given by

$$
\left(T\llcorner A)\left(f, \pi_{1}, \ldots, \pi_{m}\right):=T\left(f \chi_{A}, \pi_{1}, \ldots, \pi_{m}\right) .\right.
$$

This expression is well-defined since $T$ can be extended to a functional on tuples for which the first argument lies in $L^{\infty}(Y,\|T\|)$.

If $m \geq 1$ and $T \in \mathbf{M}_{m}(Y)$ then the boundary of $T$ is the functional

$$
\partial T\left(f, \pi_{1}, \ldots, \pi_{m-1}\right):=T\left(1, f, \pi_{1}, \ldots, \pi_{m-1}\right) .
$$

It is clear that $\partial T$ satisfies conditions (i) and (ii) in the above definition. If $\partial T$ also satisfies (iii) then $T$ is called a normal current. By convention, elements of $\mathbf{M}_{0}(Y)$ are also called normal currents.

The push-forward of $T \in \mathbf{M}_{m}(Y)$ under a Lipschitz map $\varphi$ from $Y$ to another complete metric space $Z$ is given by

$$
\varphi_{\#} T\left(g, \tau_{1}, \ldots, \tau_{m}\right):=T\left(g \circ \varphi, \tau_{1} \circ \varphi, \ldots, \tau_{m} \circ \varphi\right)
$$

for $\left(g, \tau_{1}, \ldots, \tau_{m}\right) \in \mathcal{D}^{m}(Z)$. This defines a $m$-dimensional current on $Z$. It follows directly from the definitions that $\partial\left(\varphi_{\#} T\right)=\varphi_{\#}(\partial T)$. 
We will mainly be concerned with integral currents. Let $\mathcal{H}^{m}$ denote Hausdorff $m$-measure on $Y$ and recall that an $\mathcal{H}^{m}$-measurable set $A \subset Y$ is said to be countably $\mathcal{H}^{m}$-rectifiable if there exist countably many Lipschitz maps $\varphi_{i}: B_{i} \longrightarrow Y$ from subsets $B_{i} \subset \mathbb{R}^{m}$ such that

$$
\mathcal{H}^{m}\left(A \backslash \bigcup \varphi_{i}\left(B_{i}\right)\right)=0 .
$$

An element $T \in \mathbf{M}_{0}(Y)$ is called integer rectifiable if there exist finitely many points $y_{1}, \ldots, y_{n} \in Y$ and $\theta_{1}, \ldots, \theta_{n} \in \mathbb{Z} \backslash\{0\}$ such that

$$
T(f)=\sum_{i=1}^{n} \theta_{i} f\left(y_{i}\right)
$$

for all bounded Lipschitz functions $f$. A current $T \in \mathbf{M}_{m}(Y)$ with $m \geq 1$ is said to be integer rectifiable if the following properties hold:

(i) $\|T\|$ is concentrated on a countably $\mathcal{H}^{m}$-rectifiable set and vanishes on $\mathcal{H}^{m}$-negligible Borel sets.

(ii) For any Lipschitz map $\varphi: Y \rightarrow \mathbb{R}^{m}$ and any open set $U \subset Y$ there exists $\theta \in$ $L^{1}\left(\mathbb{R}^{m}, \mathbb{Z}\right)$ such that $\varphi_{\#}(T\llcorner U)=\llbracket \theta \rrbracket$.

Integer rectifiable normal currents are called integral currents. The corresponding space is denoted by $\mathbf{I}_{m}(Y)$. If $A \subset \mathbb{R}^{m}$ is a Borel set of finite measure and finite perimeter then $\llbracket \chi_{A} \rrbracket \in \mathbf{I}_{m}\left(\mathbb{R}^{m}\right)$. Here, $\chi_{A}$ denotes the characteristic function. If $T \in \mathbf{I}_{m}(Y)$ and if $\varphi: Y \rightarrow Z$ is a Lipschitz map into another complete metric space then $\varphi_{\#} T \in \mathbf{I}_{m}(Z)$.

We close this section with a few remarks. A Lipschitz curve $c:[a, b] \rightarrow Y$ gives rise

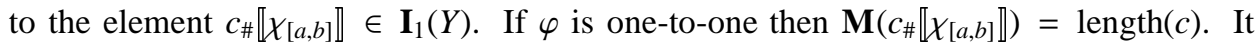
was shown in [25, Lemma 2.3] that 1-dimensional integral currents are essentially induced by (countably many) Lipschitz curves. A Lipschitz map $\varphi: D^{2} \rightarrow Y$ gives rise to the 2-dimensional integral current $S:=\varphi_{\#} \llbracket \chi_{D^{2}} \rrbracket$. If $\varphi$ is one-to-one then

$$
\frac{1}{c} \operatorname{Area}(\varphi) \leq \mathbf{M}(S) \leq c \operatorname{Area}(\varphi)
$$

for some universal $c$; if $Y$ is a Riemannian manifold then $\mathbf{M}(S)=\operatorname{Area}(\varphi)$. A singular Lipschitz chain $c=\sum m_{i} \varphi_{i}$ gives rise to the integral current $\sum m_{i} \varphi_{i \#} \llbracket \chi_{\Delta} \rrbracket$. An element $T \in \mathbf{I}_{2}(Y)$ can be thought of as a generalized singular Lipschitz chain whose boundary consists of a union of closed Lipschitz curves of finite total length.

\section{QUADRATIC ISOPERIMETRIC INEQUALITIES AND ASYMPTOTIC CONES}

A complete metric space $X$ will be said to admit a quadratic isoperimetric inequality for $\mathbf{I}_{1}(X)$ if there exists $C>0$ such that for every $T \in \mathbf{I}_{1}(X)$ with $\partial T=0$ there exists $S \in \mathbf{I}_{2}(X)$ with

$$
\mathbf{M}(S) \leq C \mathbf{M}(T)^{2} .
$$

The number $C$ will be called isoperimetric constant. The main result of this section is the following theorem which will be needed in the proof of Theorem 1.2 and which should be of independent interest.

Theorem 3.1. Let $X$ be a complete metric length space. If $X$ admits a quadratic isoperimetric inequality for $\mathbf{I}_{1}(X)$ with isoperimetric constant $C$ then every asymptotic cone $X_{\omega}$ of $X$ admits a quadratic isoperimetric inequality for $\mathbf{I}_{1}\left(X_{\omega}\right)$ with isoperimetric constant $4 C$.

For the proof of Theorem 1.2 we will actually only need Theorem 3.1 for $X$ a Carnot group endowed with a left-invariant Riemannian metric. In this case, the proof can be simplified, 
see Proposition 3.6 We believe that Theorem 3.1 should be of independent interest. As a consequence of Theorem 3.1 we obtain the following result.

Corollary 3.2. Let $X$ be a metric length space which admits a coarse homological isoperimetric inequality for curves. Then every asymptotic cone $X_{\omega}$ of $X$ admits a quadratic isoperimetric inequality for $\mathbf{I}_{1}\left(X_{\omega}\right)$.

The definition of coarse homological quadratic isoperimetric inequality for curves is given in [25]. It is a homological analog of the notion introduced in [7, III.H.2].

Proof of Corollary 3.2. By Proposition 3.2 and Lemma 2.3 of [25], $X$ has a thickening $X_{\delta}$ which is a complete metric length space admitting a quadratic isoperimetric inequality for $\mathbf{I}_{1}\left(X_{\delta}\right)$. By definition, a thickening of $X$ is a metric space which contains $X$ isometrically and which is at finite Hausdorff distance from $X$. Now, since asymptotic cones of $X_{\delta}$ and $X$ are the same, Theorem 3.1 shows that every asymptotic cone of $X$ has a quadratic isoperimetric inequality for integral 1-currents.

A special case of the above corollary is the following result.

Corollary 3.3. Let $\Gamma$ be a finitely presented group with quadratic Dehn function. Then every asymptotic cone of $\Gamma$ admits a quadratic isoperimetric inequality for integral 1currents.

It is known that every asymptotic cone of a finitely presented group with quadratic Dehn function is simply connected, [19]. However, many groups have simply connected asymptotic cones even though their Dehn function is not quadratic; for example, the asymptotic cone of any Carnot group is simply connected. Theorem 3.1 does not yield simple connectedness but instead gives strong metric information about the asymptotic cones.

We turn to the proof of Theorem 3.1 for which we need two simple lemmas.

Lemma 3.4. Let $Y$ be a complete metric length space and $D>0$ and suppose $Y$ satisfies the following condition: for every closed Lipschitz curve $c:[0,1] \rightarrow Y$ and every finite partition $0=t_{1}<t_{2}<\cdots<t_{m}=1$ of $[0,1]$ there exist a Lipschitz curve $c^{\prime}:[0,1] \rightarrow Y$ and $S \in \mathbf{I}_{2}(Y)$ satisfying $c^{\prime}\left(t_{i}\right)=c\left(t_{i}\right)$ and

$$
\text { length }\left(\left.c^{\prime}\right|_{\left[t_{i}, t_{i+1}\right]}\right) \leq \operatorname{length}\left(\left.c\right|_{\left[t_{i}, t_{i+1}\right]}\right)
$$

for all $i$, as well as $\partial S=c_{\#}^{\prime} \llbracket \chi_{[0,1]} \rrbracket$ and

$$
\mathbf{M}(S) \leq D \text { length }(c)^{2} .
$$

Then $Y$ admits a quadratic isoperimetric inequality for $\mathbf{I}_{1}(Y)$ with isoperimetric constant $2 D$.

Proof. Let $c:[0,1] \rightarrow Y$ be a closed Lipschitz curve and set $T:=c_{\#} \llbracket \chi_{[0,1]} \rrbracket$. We will find, for each $n \geq 0$, closed Lipschitz curves $c_{n, i}:[0,1] \rightarrow Y, i=1, \ldots, 8^{n}$, and $S_{n} \in \mathbf{I}_{2}(Y)$ such that

$$
\text { length }\left(c_{n, i}\right) \leq \frac{1}{4^{n}} \text { length }(c) \quad \text { and } \quad \mathbf{M}\left(S_{n}\right) \leq \frac{1}{2^{n-1}} D \text { length }(c)^{2},
$$

as well as

$$
T=\partial\left(S_{0}+\cdots+S_{n}\right)+\sum_{i=1}^{8^{n}} c_{n, i \#} \llbracket \chi_{[0,1]} \rrbracket .
$$


For $n=0$ we simply set $c_{0,1}:=c$ and $S_{0}:=0$. Suppose we have found $S_{0}, \ldots, S_{n-1}$ and $c_{n-1, i}$ for some $n \geq 1$ with the properties listed above. In order to construct $c_{n, j}$ and $S_{n}$, we proceed as follows. First, fix $i:=1$ and choose $0=t_{1}<t_{2}<\cdots<t_{9}=1$ such that

$$
\text { length }\left(c_{n-1, i} \mid\left[t_{j}, t_{j+1}\right]\right)=\frac{1}{8} \text { length }\left(c_{n-1, i}\right)
$$

for $j=1, \ldots, 8$. By assumption, there exist a closed Lipschitz curve $c^{\prime}:[0,1] \rightarrow Y$ and $S \in \mathbf{I}_{2}(Y)$ such that $c^{\prime}\left(t_{j}\right)=c_{n-1, i}\left(t_{j}\right)$ and

$$
\text { length }\left(\left.c^{\prime}\right|_{\left[t_{j}, t_{j+1}\right]}\right) \leq \text { length }\left(c_{n-1, i} \mid\left[t_{j}, t_{j+1}\right]\right)
$$

for all $j$, as well as $\partial S=c_{\#}^{\prime} \llbracket \chi_{[0,1]} \rrbracket$ and

$$
\mathbf{M}(S) \leq D \text { length }\left(c_{n-1, i}\right)^{2} .
$$

Set $S_{n, 1}:=S$ and, for $j=1, \ldots, 8$, let $c_{n, j}$ be the concatenation of $c_{n-1, i \mid\left[t_{j}, t_{j+1}\right]}$ with $\left(\left.c^{\prime}\right|_{\left[t_{j}, t_{j+1}\right]}\right)^{-}$. It is clear that

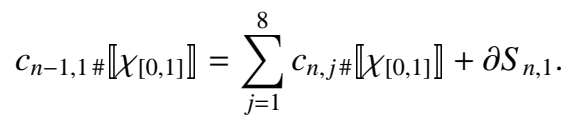

Now, do the same for $i=2, \ldots, 8^{n-1}$ in order to obtain, after relabeling indices, curves $c_{n, j}, j=1, \ldots, 8^{n}$, and currents $S_{n, j}, j=1, \ldots, 8^{n-1}$. Clearly, we have length $\left(c_{n, j}\right) \leq$ $4^{-n}$ length $(c)$ and, for

$$
S_{n}:=S_{n, 1}+\cdots+S_{n, 8^{n-1}},
$$

we have

$$
\mathbf{M}\left(S_{n}\right) \leq D \sum_{i=1}^{8^{n-1}} \text { length }\left(c_{n-1, i}\right)^{2} \leq 8^{n-1} D \cdot\left(\frac{1}{4^{n-1}} \text { length }(c)\right)^{2}=\frac{1}{2^{n-1}} D \text { length }(c)^{2} .
$$

Finally, the fact that

$$
T=\partial\left(S_{0}+\cdots+S_{n}\right)+\sum_{i=1}^{8^{n}} c_{n, i \#} \llbracket \chi_{[0,1]} \rrbracket
$$

follows from (3). This proves the existence of $c_{n, i}$ and $S_{n}$ with the properties stated above. Now, set $S:=\sum_{n=1}^{\infty} S_{n}$ and note that $S$ is an integer rectifiable 2-current satisfying

$$
\mathbf{M}(S) \leq \sum_{n=1}^{\infty} \mathbf{M}\left(S_{n}\right) \leq 2 D \text { length }(c)^{2} .
$$

It is not difficult to show that $\partial S=T$. Indeed, view $Y$ as a subset of $Y^{\prime}:=l^{\infty}(Y)$. It is well-known, see [23], that $Y^{\prime}$ has a quadratic isoperimetric inequality for $\mathbf{I}_{1}\left(Y^{\prime}\right)$. Let $D^{\prime}$ be the isoperimetric constant. For each $n$ and $i=1, \ldots, 8^{n}$, there thus exists $Q_{n, i} \in \mathbf{I}_{2}\left(Y^{\prime}\right)$ with $\partial Q_{n, i}=c_{n, i} \llbracket \llbracket \chi_{[0,1]} \rrbracket$ and such that $\mathbf{M}\left(Q_{n, i}\right) \leq D^{\prime}$ length $\left(c_{n, i}\right)^{2}$. Set $Q_{n}:=Q_{n, 1}+\cdots+Q_{n, 8^{n}}$, note that $Q_{n} \in \mathbf{I}_{2}\left(Y^{\prime}\right)$,

$$
\mathbf{M}\left(Q_{n}\right) \leq D^{\prime} \sum_{i=1}^{8^{n}} \text { length }\left(c_{n, i}\right)^{2} \leq \frac{1}{2^{n}} D^{\prime} \text { length }(c)^{2},
$$

and $T=\partial\left(S_{1}+\cdots+S_{n}\right)+\partial Q_{n}$. Since $Q_{n}$ converges to 0 in mass and $S_{1}+\cdots+S_{n}$ converges in mass to $S$, their boundaries converge weakly to 0 and $\partial S$, respectively. This shows that indeed $\partial S=T$. Since $S$ satisfies (4) and since $c$ was arbitrary, Lemma 2.3 of [25] shows that $Y$ admits a quadratic isoperimetric inequality for $\mathbf{I}_{1}(Y)$ with isoperimetric constant $2 D$. 
Lemma 3.5. Let $Z$ be a compact metric space, $m \in \mathbb{N}$, and $\omega$ a non-principal ultrafilter on $\mathbb{N}$. Suppose $A_{n} \subset Z$ are closed subsets and $a_{n}^{1}, \ldots, a_{n}^{m} \in A_{n}$ for $n \geq 1$. Then there exists a subsequence $\left(A_{n_{j}}\right)$ such that $A_{n_{j}}$ converges in the Hausdorff distance to a closed subset of

$$
A:=\left\{\lim _{\omega} a_{n}: a_{n} \in A_{n} \text { for every } n\right\}
$$

and $a_{n_{j}}^{i} \rightarrow \lim _{\omega} a_{n}^{i}$ as $j \rightarrow \infty$, for all $i=1, \ldots, m$.

Proof. Since $Z$ is compact, there exist integers $m \leq m_{1}<m_{2}<\ldots$ and, for each $n \in \mathbb{N}$, a sequence $\left(a_{n}^{j}\right)_{j \geq m+1} \subset A_{n}$ of points such that $\left\{a_{n}^{j}: j=1, \ldots, m_{i}\right\}$ is $2^{-i}$-dense in $A_{n}$. For each $i$ set $a^{i}:=\lim _{\omega} a_{n}^{i}$, and denote by $C$ the closure of $\left\{a^{i}: i \in \mathbb{N}\right\}$; clearly $C \subset A$. Define for each $j \in \mathbb{N}$

$$
\Omega_{j}:=\left\{n: d\left(a_{n}^{i}, a^{i}\right) \leq 2^{-j} \text { for } i=1, \ldots, m_{j}\right\}
$$

and note that $\Omega_{1} \supset \Omega_{2} \supset \ldots$ and that $\omega\left(\Omega_{j}\right)=1$ for every $j$; in particular, $\Omega_{j}$ is not finite. Choose $n_{1}<n_{2}<\ldots$ with $n_{j} \in \Omega_{j}$ for all $j$. It follows that $A_{n_{j}}$ converges in the Hausdorff sense to $C$. Furthermore, we have that $a_{n_{j}}^{i}$ converges to $a^{i}$ as $j \rightarrow \infty$ for each $i$ and, in particular, for $i=1, \ldots, m$. This concludes the proof.

We now use the lemmas above to prove the main theorem of this section.

Proof of Theorem 3.1 Let $\omega$ be a non-principal ultrafilter on $\mathbb{N}$, let $p_{n} \in X$, and $r_{n}>0$ with $r_{n} \rightarrow \infty$. Let $X_{\omega}$ be the asymptotic cone associated with the pointed sequence $\left(X, r_{n}^{-1} d, p_{n}\right)$ and $\omega$. Denote by $X_{n}$ the space $X$ endowed with the metric $d_{n}:=r_{n}^{-1} d$. Let $c:[0,1] \rightarrow X_{\omega}$ be a closed Lipschitz curve and $0=t_{1}<t_{2}<\cdots<t_{m}=1$ a partition of $[0,1]$. Set $x^{i}:=c\left(t_{i}\right)$. Then $x^{i}=\left[\left(x_{n}^{i}\right)\right]$ for some $x_{n}^{i} \in X$ with

$$
\sup _{n} d_{n}\left(x_{n}^{i}, p_{n}\right)<\infty
$$

and we may assume that $x_{n}^{m}=x_{n}^{1}$ for all $n$. Let $c_{n}:[0,1] \rightarrow X_{n}$ be a Lipschitz curve such that for all $i$ we have $c_{n}\left(t_{i}\right)=x_{n}^{i}$ and $\left.c_{n}\right|_{\left[t_{i}, t_{i+1}\right]}$ is parametrized proportional to arc-length with

Note that

$$
\text { length }\left(\left.c_{n}\right|_{\left[t_{i}, t_{i+1}\right]}\right) \leq d_{n}\left(x_{n}^{i}, x_{n}^{i+1}\right)+\frac{1}{m n} \text {. }
$$

$$
\sup _{n} \operatorname{Lip}\left(c_{n}\right)<\infty
$$

and that there exists $R>0$ such that $c_{n}$ has image in the ball $B_{X_{n}}\left(p_{n}, R\right)$ for all $n$. It follows in particular that $\sup _{n}$ length $\left(c_{n}\right)<\infty$. Set $T_{n}:=c_{n \#} \llbracket \chi_{[0,1]} \rrbracket$ and note that $T_{n} \in \mathbf{I}_{1}\left(X_{n}\right)$ satisfies $\partial T_{n}=0$ and $\mathbf{M}\left(T_{n}\right) \leq$ length $\left(c_{n}\right)+1 / n$. Set $D:=2 C$ where $C$ is the isoperimetric constant for $X$. By Lemma 3.4 in [23], see also Theorem 10.6 in [3], there exists $S_{n} \in$ $\mathbf{I}_{2}\left(X_{n}\right)$ such that $\partial S_{n}=T_{n}$ and

$$
\mathbf{M}\left(S_{n}\right) \leq D \mathbf{M}\left(T_{n}\right)^{2}
$$

and such that the sequence $\left(Y_{n}\right)$ of metric spaces $Y_{n}$ given by

$$
Y_{n}:=\left(\operatorname{spt} S_{n} \cup c_{n}([0,1]) \cup\left\{p_{n}\right\}, d_{n}\right)
$$

is uniformly compact in the sense of Gromov. It thus follows from Gromov's compactness theorem [10] that there exist a compact metric space $\left(Z, d_{Z}\right)$ and isometric embeddings $\varphi_{n}: Y_{n} \rightarrow Z$ for all $n$. Set $A_{n}:=\varphi_{n}\left(Y_{n}\right)$ and $a_{n}^{i}:=\varphi_{n}\left(c_{n}\left(t_{i}\right)\right)$ for $i=1, \ldots, m$. By Lemma 3.5 there exists a subsequence $\left(A_{n_{j}}\right)$ such that $A_{n_{j}}$ converges in the Hausdorff sense to a closed subset of $A:=\left\{\lim _{\omega} a_{n}: a_{n} \in A_{n}\right.$ for all $\left.n\right\}$ and $a_{n_{j}}^{i}$ converges to $a^{i}:=\lim _{\omega} a_{n}^{i}$ for every $i$. After possibly passing to a further subsequence, we may assume that $\psi_{j}:=\varphi_{n_{j}} \circ c_{n_{j}}$ converges uniformly to a Lipschitz curve $\psi:[0,1] \rightarrow Z$ and that $\varphi_{n_{j} \#} S_{n_{j}}$ converges weakly 
to some $S^{\prime} \in \mathbf{I}_{2}(Z)$. The second assertion is a consequence of the closure and compactness theorems for integral currents in compact metric spaces, see Theorems 5.2 and 8.5 in [3].

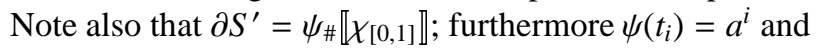

$$
\text { length }\left(\psi_{j}\right) \leq \frac{1}{n_{j}}+\sum_{i=1}^{m-1} d_{Z}\left(a_{n_{j}}^{i}, a_{n_{j}}^{i+1}\right)
$$

Since $a_{n_{j}}^{i} \rightarrow a^{i}$ and $d_{Z}\left(a^{i}, a^{i+1}\right)=d_{\omega}\left(x^{i}, x^{i+1}\right)$, we conclude

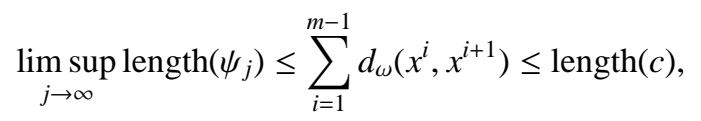

hence also length $(\psi) \leq$ length $(c)$ and

$$
\mathbf{M}\left(S^{\prime}\right) \leq D \liminf _{n \rightarrow \infty} \mathbf{M}\left(\varphi_{n_{j} \#} T_{n}\right)^{2} \leq \liminf _{n \rightarrow \infty} \text { length }\left(\psi_{j}\right)^{2} \leq D \text { length }(c)^{2} .
$$

Since $A_{n_{j}}$ converges in the Hausdorff sense to a closed subset of $A$ we furthermore have

$$
\psi([0,1]) \cup \operatorname{spt} S^{\prime} \subset A .
$$

Let now $\Lambda: A \rightarrow X_{\omega}$ be the isometric embedding defined by $\Lambda(a):=\left[\left(y_{n}\right)\right]$, where $\left(y_{n}\right) \subset X$ is a sequence with $y_{n} \in Y_{n}$ and $a=\lim _{\omega} \varphi_{n}\left(y_{n}\right)$. It is not difficult to show that $\Lambda$ is well-defined and is an isometric embedding. Furthermore, the Lipschitz curve $c^{\prime}:=\Lambda \circ \psi$ satisfies $c^{\prime}\left(t_{i}\right)=x^{i}=c\left(t_{i}\right)$ and

$$
\text { length }\left(c^{\prime}\right) \leq \text { length }(c) \text {. }
$$

We set $S:=\Lambda_{\#} S^{\prime}$ and note that $\partial S=c_{\#}^{\prime} \llbracket \chi_{[0,1]} \rrbracket$ and $\mathbf{M}(S) \leq D$ length $(c)^{2}$. In view of Lemma 3.4 the proof is complete.

As mentioned above, in the special case when $X$ is a Carnot group endowed with a leftinvariant Riemannian metric, the proof of Theorem 3.1 can be simplified. In fact, we can prove an analog for higher-dimensional cycles. Let $m \geq 1$. A complete metric space $X$ is said to admit an isoperimetric inequality of Euclidean type for $\mathbf{I}_{m}(X)$ if there exists $C>0$ such that for every $T \in \mathbf{I}_{m}(X)$ with $\partial T=0$ there exists $S \in \mathbf{I}_{m+1}(X)$ with

$$
\mathbf{M}(S) \leq C \mathbf{M}(T)^{\frac{m+1}{m}} .
$$

If the above holds only for $T$ with spt $T$ compact, then $X$ will be said to admit an isoperimetric inequality of Euclidean type for compactly supported integral $m$-currents.

Let $G$ be a Carnot group and $d_{0}$ the metric on $G$ coming from a left-invariant Riemannian metric, and let $d_{c}$ be the associated Carnot-Carathéodory metric.

Proposition 3.6. Let $m \geq 1$. If $X:=\left(G, d_{0}\right)$ admits an isoperimetric inequality of Euclidean type for $\mathbf{I}_{m}(X)$ then $Y:=\left(G, d_{c}\right)$ admits an isoperimetric inequality of Euclidean type for compactly supported integral m-currents.

Note that in the case $m=1$, Proposition 3.6 together with [25, Lemma 2.3] yield a quadratic isoperimetric inequality for $\mathbf{I}_{1}(Y)$, that is, also for integral 1-currents whose supports are not compact.

Proof. Let $T \in \mathbf{I}_{m}(Y)$ with spt $T$ compact and $\partial T=0$. Denote by $\varphi: Y \rightarrow X$ the identity map and note that $\varphi$ is 1-Lipschitz. For each $n \geq 1$ define $T_{n}:=\left(\varphi \circ \delta_{n}\right)_{\#} T \in \mathbf{I}_{m}(X)$. Clearly, we have $\partial T_{n}=0$ and $\mathbf{M}\left(T_{n}\right) \leq n^{m} \mathbf{M}(T)$. By Lemma 3.4 in [23] (see also Theorem 10.6 in [3]), there exists $S_{n} \in \mathbf{I}_{m+1}(X)$ such that $\partial S_{n}=T_{n}$,

$$
\mathbf{M}\left(S_{n}\right) \leq C \mathbf{M}\left(T_{n}\right)^{\frac{m+1}{m}} \leq C n^{m+1} \mathbf{M}(T)^{\frac{m+1}{m}},
$$


and

$$
\left\|S_{n}\right\|(B(x, r)) \geq C^{\prime} r^{m+1}
$$

for all $x \in \operatorname{spt} S_{n}$ and $0 \leq r \leq d\left(x\right.$, spt $\left.T_{n}\right)$. Here, $C$ and $C^{\prime}$ are constants only depending on the isoperimetric constant for $\mathbf{I}_{m}(X)$. It follows that there exists $L$ such that $\operatorname{spt} S_{n} \subset$ $B(e, n L)$ for every $n$, where $e$ is the identity in $G$. Define metric spaces by

$$
Y_{n}:=\left(\operatorname{spt} S_{n}, \frac{1}{n} d_{0}\right) \text {. }
$$

It follows that $\left(Y_{n}\right)$ is uniformly compact and thus, by Gromov's compactness theorem, there exists a compact metric space $Z$ and isometric embeddings $\psi_{n}: Y_{n} \rightarrow Z$ for every $n$. Define subsets $A_{n}:=\psi_{n}\left(\operatorname{spt} S_{n}\right)$ of $Z$ and maps $\varrho_{n}:=\psi_{n} \circ \varphi \circ \delta_{n}:\left(\operatorname{spt} T, d_{c}\right) \rightarrow Z$. After possibly passing to a subsequence, we may assume that $\left(A_{n}\right)$ converges to a closed subset $A \subset Z$ in the Hausdorff sense and $\varrho_{n}$ converges uniformly to a 1-Lipschitz map $\varrho:\left(\operatorname{spt} T, d_{c}\right) \rightarrow Z$. After possibly passing to a further subsequence, we may assume by the compactness and closure theorems (see Theorems 5.2 and 8.5 in [3]) that $\psi_{n \#} S_{n}$ converges weakly to some $\hat{S} \in \mathbf{I}_{m+1}(Z)$. Let $\omega$ be a non-principal ultrafilter on $\mathbb{N}$ and define a map $\eta: A \rightarrow Y$ as follows. Given $a \in A$, let $x_{n} \in \operatorname{spt} S_{n}$ such that $\psi_{n}\left(x_{n}\right) \rightarrow a$, and define

$$
\eta(a):=\lim _{\omega} \delta_{\frac{1}{n}}\left(x_{n}\right)
$$

It is not difficult to show that $\eta$ is well-defined, an isometric embedding, and satisfies $\eta \circ \varrho=\operatorname{id}_{\text {spt } T}$. Set $S:=\eta_{\#} \hat{S}$ and note that $\partial S=T$ as well as

$$
\mathbf{M}(S) \leq \liminf _{n \rightarrow \infty} \frac{1}{n^{m+1}} \mathbf{M}\left(S_{n}\right) \leq C \mathbf{M}(T)^{\frac{m+1}{m}} .
$$

This concludes the proof.

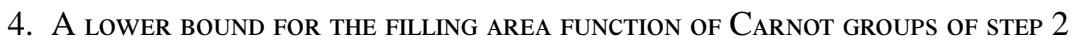

In this section we prove new lower bounds for the filling area function of the central product of certain Carnot groups of step 2. The main result is Theorem 4.3. which we will use to prove Theorem 1.2 .

Let $G$ be a Carnot group of step 2 with grading $\mathfrak{g}=V_{1} \oplus V_{2}$ of its Lie algebra $\mathfrak{g}$ and Lie bracket $[\cdot, \cdot]$. If $U$ is a subspace of $V_{2}$ then $[\cdot, \cdot]$ naturally induces a Lie bracket $[\cdot, \cdot]_{U}$ on $\mathfrak{g}_{U}=V_{1} \oplus V_{2}^{\prime}$, where $V_{2}^{\prime}:=V_{2} / U$ is the quotient space. Let $G_{U}$ be the Carnot group whose Lie algebra is $\mathfrak{g}_{U}$. Note that every connected and simply connected nilpotent Lie group of step 2 is of the form $G_{U}$ with $\mathfrak{g}=V_{1} \oplus V_{2}$ a free nilpotent Lie algebra of step 2 and $U \subset V_{2}$ a suitable subspace. We will use the following terminology.

Definition 4.1. Let $m \geq 1$. A non-trivial subspace $U$ of $V_{2}$ is called $m$-inaccessible if there exists a proper subspace $U^{\prime}$ of $U$, possibly $U^{\prime}=\{0\}$, such that if $v_{1}, \ldots, v_{m}, w_{1}, \ldots, w_{m} \in V_{1}$ then the vector $\left[v_{1}, w_{1}\right]+\cdots+\left[v_{m}, w_{m}\right]$ is contained in $U$ if and only it is contained in $U^{\prime}$.

It is not difficult to give examples of graded nilpotent Lie algebras $\mathfrak{g}=V_{1} \oplus V_{2}$ such that $V_{2}$ has a non-trivial $m$-inaccessible subspace.

Example 4.2. Let $k, m$ satisfy $k \geq 2(m+1)$ and let $\mathfrak{g}=V_{1} \oplus V_{2}$ be the free nilpotent Lie algebra of step 2 with $\operatorname{dim} V_{1}=k$. Then there exist a basis $\left\{e_{1}, \ldots, e_{k}\right\}$ of $V_{1}$ and a basis $\left\{e_{i, j}: 1 \leq i<j \leq k\right\}$ of $V_{2}$ such that the Lie bracket on $\mathrm{g}$ satisfies $\left[e_{i}, e_{j}\right]=e_{i, j}$ whenever $i<j$. It is straightforward to check that the one-dimensional subspace

$$
U:=\operatorname{span}\left\{e_{1,2}+e_{3,4}+\cdots+e_{2 m+1,2 m+2}\right\}
$$


of $V_{2}$ is $m$-inaccessible. One may in fact take $U^{\prime}=\{0\}$. Note that here $\mathfrak{g}_{U}$ has a basis with rational structure constants.

More generally, if $\mathfrak{g}=V_{1} \oplus V_{2}$ is a stratified nilpotent Lie algebra of step 2 with

$$
\operatorname{dim} V_{2}>\left(2 \operatorname{dim} V_{1}-1\right) m
$$

then $V_{2}$ possesses an $m$-inaccessible subspace $U$; furthermore, $U$ may be chosen such that $\mathfrak{g}_{U}$ has a basis with rational structure constants. Indeed, the subset $C_{m} \subset V_{2}$ given by

$$
C_{m}:=\left\{\left[v_{1}, w_{1}\right]+\cdots+\left[v_{m}, w_{m}\right]: v_{i}, w_{i} \in V_{1}\right\}
$$

is a cone containing 0 and is the image of a smooth map $\psi:\left(\mathbb{R} \times S^{k} \times S^{k}\right)^{m} \rightarrow V_{2}$, where $k=\operatorname{dim} V_{1}-1$. Consequently, $C_{m}$ has Hausdorff dimension at most $\left(2 \operatorname{dim} V_{1}-1\right) m$ and hence $C_{m} \neq V_{2}$ as soon as (5) holds. Clearly, for $v \in V_{2} \backslash C_{m}$, the subspace $U:=\operatorname{span}\{v\}$ is $m$-inaccessible.

We turn to the main result of this section.

Theorem 4.3. Let $G$ be a Carnot group of step 2 with grading $\mathfrak{g}=V_{1} \oplus V_{2}$ of its Lie algebra. Suppose $U \subset V_{2}$ is an m-inaccessible subspace where $m \geq 1$. Let $G_{U}$ be the Carnot group of step 2 whose Lie algebra is $\mathfrak{g}_{U}=V_{1} \oplus V_{2}^{\prime}$, where $V_{2}^{\prime}:=V_{2} / U$, and let

$$
H:=G_{U} \times_{Z} \cdots \times_{Z} G_{U}
$$

be the central product of $m$ copies of $G_{U}$. Then, endowed with a left-invariant Riemannian metric, $H$ has filling area function which grows strictly faster than quadratically:

$$
\frac{\mathrm{FA}(r)}{r^{2}} \rightarrow \infty \quad \text { as } r \rightarrow \infty .
$$

For the definition of $\mathrm{FA}(r)$ see Section 2.1. Recall that the central product of $m$ copies of a group $\Gamma$ is the quotient of the $m$-fold direct product of $\Gamma$ by the normal subgroup $N$ of tuples $\left(g_{1}, \ldots, g_{m}\right)$ with $g_{i} \in[\Gamma, \Gamma]$ and $g_{1} \cdots g_{m}=e$, where $e$ is the identity element of $\Gamma$. In order to prove Theorem 4.3 we will actually show that there exists a closed Lipschitz curve in $\left(H, d_{c}\right)$ which does not bound an integral 2-current in $\left(H, d_{c}\right)$, where $d_{c}$ is the Carnot-Carathéodory distance associated with the left-invariant Riemannian metric. The super-quadratic growth of FA $(r)$ then follows from Theorem 3.1 or Proposition 3.6 From Theorem 4.3 and the fact that central products of finitely generated nilpotent groups of step 2 have Dehn function bounded above by $n^{2} \log n$, see [16, 27], we obtain:

Corollary 4.4. Let $G_{U}$ and $H$ be as in Theorem 4.3 for some $m \geq 2$, and suppose the Lie algebra of $G_{U}$ has a basis with rational structure constants. Then the filling area and Dehn functions of $H$ satisfy

$$
r^{2} \varrho(r) \leq \mathrm{FA}(r) \leq \mathrm{FA}_{0}(r) \leq C r^{2} \log r
$$

for all $r \geq 2$, where $\varrho$ is a function satisfying $\varrho(r) \rightarrow \infty$ as $r \rightarrow \infty$.

Note that if $\mathfrak{g}$ and $U$ are as in Example 4.2 for some $m \geq 2$ then, in particular, $G_{U}$ and its $m$-th central power $H=G_{U} \times_{Z} \cdots \times_{Z} G_{U}$ satisfy the hypotheses of Corollary 4.4 Consequently, the filling area and Dehn functions of $H$ satisfy (6). This answers in the negative the question raised in [27] whether the Carnot group $\Gamma=G_{U} \times{ }_{Z} G_{U}$, where $g$ and $U$ are as in Example 4.2 with $k=10$ and $m=2$, has quadratic Dehn function.

Proof of Corollary 4.4. The lower bound for FA( $r$ ) in (6) comes from Theorem 4.3. In order to prove the upper bound for $\mathrm{FA}_{0}(r)$ let $\mathfrak{g}_{U}$ denote the Lie algebra of $G_{U}$ with grading $\mathfrak{g}_{U}=V_{1} \oplus V_{2}^{\prime}$. Since $\mathfrak{g}_{U}$ has a basis with rational structure constants, there exists a basis 
of vectors in $V_{1}$ which generate a lattice $\Gamma$ in $G_{U}$. Now, there exists an injective homomorphism from the central product $\Gamma^{\prime}:=\Gamma \times_{Z} \cdots \times_{Z} \Gamma$ of $m$ copies of $\Gamma$ to $H$ whose image is a lattice in $H$. Therefore, the Dehn function $\delta_{\Gamma^{\prime}}(n)$ of $\Gamma^{\prime}$ satisfies

$$
\delta_{\Gamma^{\prime}}(n) \sim \mathrm{FA}_{0}(n) .
$$

Since $\Gamma^{\prime}$ is a central product of $m \geq 2$ copies of a nilpotent group of step 2, we have that $\delta_{\Gamma^{\prime}}(n) \leq n^{2} \log n$. This was first proved by Ol'shanskii-Sapir using the techniques of [16], see the remark on page 927 in [16]. More recently, Young gave a proof of this in [27] using different methods. It follows that there exists a suitable constant $C$ such that $\mathrm{FA}_{0}(r)$ satisfies

$$
\mathrm{FA}_{0}(r) \leq C r^{2} \log r
$$

for all $r \geq 2$. This concludes the proof.

Theorem 1.2 is a direct consequence of (the proof) of Corollary 4.4 Indeed, for the finitely generated $\Gamma^{\prime}:=\Gamma \times_{Z} \cdots \times_{Z} \Gamma$ appearing in the above proof, we have

$$
n^{2} \varrho(n) \leq \delta_{\Gamma}(n) \leq n^{2} \log n
$$

for some function $\varrho$ with $\varrho(n) \rightarrow \infty$ as $n \rightarrow \infty$.

4.1. The proof of Theorem 4.3, In the proof of the theorem it will often be useful to identify a given Carnot group $G$ via the exponential map with $(\mathfrak{g}, *)$, where $\mathfrak{g}$ is the Lie algebra of $G$ (viewed as a vector space) and $*$ is the multiplication on $g$ given by the BakerCampbell-Hausdorff formula. The Lie algebra of $(\mathfrak{g}, *)$ is $\mathfrak{g}$ and the exponential map is simply the identity map on $\mathrm{g}$. If $G$ is of step 2 then $*$ is given by

$$
v * v^{\prime}=v+v^{\prime}+\frac{1}{2}\left[v, v^{\prime}\right]
$$

Proof of Theorem 4.3 Let $G, \mathfrak{g}=V_{1} \oplus V_{2}, U, \mathfrak{g}_{U}, G_{U}$, and $H$ satisfy the hypotheses of the theorem. Suppose $U^{\prime} \subset U$ is as in Definition 4.1 and let $U^{\prime \prime} \subset U$ be a subspace complementary to $U^{\prime}$, thus $U=U^{\prime}+U^{\prime \prime}$ and $U^{\prime} \cap U^{\prime \prime}=\{0\}$. Let $u_{0} \in U^{\prime \prime}$ with $u_{0} \neq 0$ and choose $v_{1}, \ldots, v_{2 k} \in V_{1}$ with

$$
u_{0}=\left[v_{1}, v_{2}\right]+\left[v_{3}, v_{4}\right]+\cdots+\left[v_{2 k-1}, v_{2 k}\right] \text {, }
$$

where $[\cdot, \cdot]$ is the Lie bracket on $\mathfrak{g}$. Define, for every $j=1, \ldots, k$, a piecewise affine curve $c_{1}^{j}:[0,4] \rightarrow V_{1}$ by

$$
c_{1}^{j}(t)=\left\{\begin{array}{cc}
t v_{2 j-1} & 0 \leq t<1 \\
v_{2 j-1}+(t-1) v_{2 j} & 1 \leq t<2 \\
(3-t) v_{2 j-1}+v_{2 j} & 2 \leq t<3 \\
(4-t) v_{2 j} & 3 \leq t \leq 4,
\end{array}\right.
$$

and let $c_{1}:[0,1] \rightarrow V_{1}$ be the concatenation of the curves $c_{1}^{j}, j=1, \ldots, k$, parametrized on $[0,1]$. Clearly, $c_{1}$ is closed and piecewise affine. Define $\hat{c}_{2}:[0,1] \rightarrow V_{2}$ by

$$
\hat{c}_{2}(t):=\frac{1}{2} \int_{0}^{t}\left[c_{1}(s), \dot{c}_{1}(s)\right] d s
$$

and observe that $\hat{c}_{2}(1)=u_{0}$. Let now

$$
W_{1}:=V_{1} \oplus \cdots \oplus V_{1}
$$

be the direct sum of $m$ copies of $V_{1}$. Define quotient spaces $W_{2}:=V_{2} / U$ and $W_{2}^{\prime}:=V_{2} / U^{\prime}$ and denote by $P: V_{2} \rightarrow W_{2}$ and $P^{\prime}: V_{2} \rightarrow W_{2}^{\prime}$ the natural projections. The Lie bracket 
$[\cdot, \cdot]$ on $g$ gives rise to the bilinear map $W_{1} \times W_{1} \rightarrow V_{2}$, denoted by the same symbol $[\cdot, \cdot]$, defined by

$$
\left[w, w^{\prime}\right]=\left[v_{1}, v_{1}^{\prime}\right]+\cdots+\left[v_{m}, v_{m}^{\prime}\right],
$$

where $w, w^{\prime} \in W_{1}$ are of the form $w=v_{1}+\cdots+v_{m}$ and $w^{\prime}=v_{1}^{\prime}+\cdots+v_{m}^{\prime}$ with $v_{i}$ and $v_{i}^{\prime}$ in the $i$-th copy of $V_{1}$. Clearly, $H$ has Lie algebra $\mathfrak{h}=W_{1} \oplus W_{2}$ with Lie bracket

$$
\left[w_{1}+w_{2}, w_{1}^{\prime}+w_{2}^{\prime}\right]_{\mathfrak{h}}:=P\left(\left[w_{1}, w_{1}^{\prime}\right]\right),
$$

where $w_{i}, w_{i}^{\prime} \in W_{i}$ for $i=1,2$. It follows from the $m$-inaccessibility of $U$ that for any $w_{1}, w_{1}^{\prime} \in W_{1}$ we have

$$
\left[w_{1}, w_{1}^{\prime}\right]_{\mathfrak{h}}=0 \quad \text { if and only if } \quad P^{\prime}\left(\left[w_{1}, w_{1}^{\prime}\right]\right)=0 .
$$

Identifying $V_{1}$ with the first of the $m$ copies of $V_{1}$ in $W_{1}$, we may view $c_{1}$ as a curve in $W_{1}$. Define a curve $c:=[0,1] \rightarrow H$ by $c:=c_{1}+P \circ \hat{c}_{2}$ and note that $c(1)=c_{1}(1)+P\left(u_{0}\right)=0$. Let $\|\cdot\|$ be a Euclidean norm on $W_{1}$ and let $d_{0}$ be the distance coming from a left-invariant Riemannian metric on $H$ which, restricted to $W_{1} \subset T_{0} H$, induces $\|\cdot\|$. Let furthermore $d_{c}$ be the Carnot-Carathéodory metric on $H$ associated with $\|\cdot\|$. Set $Y:=\left(H, d_{c}\right)$. Clearly, $c$ is a closed Lipschitz curve in $Y$. Set $T:=c_{\#} \llbracket \chi_{[0,1]} \rrbracket$. We claim that there does not exist $S \in \mathbf{I}_{2}(Y)$ with $\partial S=T$. We argue by contradiction and assume such an $S \in \mathbf{I}_{2}(Y)$ with $\partial S=T$ exists. By [3, Theorem 4.5], $S$ is of the form

$$
S=\sum_{i=1}^{\infty} \varphi_{i \#} \llbracket \theta_{i} \rrbracket
$$

for some Lipschitz maps $\varphi_{i}: K_{i} \rightarrow Y$ with $K_{i} \subset \mathbb{R}^{2}$ compact and $\theta_{i} \in L^{1}\left(K_{i}, \mathbb{Z}\right)$. Denote by $\pi: Y \rightarrow\left(W_{1},\|\cdot\|\right)$ the projection given by $\pi(w):=w_{1}$ for $w=w_{1}+w_{2}$ with $w_{i} \in W_{i}, i=1,2$. As above, $H$ is identified with $W_{1} \oplus W_{2}$. It is straightforward to check that $\pi$ is 1-Lipschitz and Pansu-differentiable at every point $y \in Y$ with Pansu-differential $d^{P} \pi(y): H \rightarrow W_{1}$ given by

$$
d^{P} \pi(x)(w)=w_{1}
$$

for $w=w_{1}+w_{2}$ with $w_{i} \in W_{i}, i=1,2$. Set $\bar{S}:=\pi_{\#} S$ and note that $\bar{S} \in \mathbf{I}_{2}\left(\left(W_{1},\|\cdot\|\right)\right)$ with

$$
\partial \bar{S}=\pi_{\#} T=c_{1 \#} \llbracket \chi_{[0,1]} \rrbracket
$$

furthermore

$$
\bar{S}=\sum_{i=1}^{\infty} \psi_{i \# \Perp} \llbracket \theta_{i} \rrbracket
$$

where $\psi_{i}:=\pi \circ \varphi_{i}$. Fix $i$. We claim that at almost every $x \in K_{i}$

$$
\left[d_{x} \psi_{i}(u), d_{x} \psi_{i}(v)\right]_{\mathfrak{h}}=0
$$

for all $u, v \in \mathbb{R}^{2}$, where $d_{x} \psi_{i}$ is the classical derivative (which exists almost everywhere by Rademacher's theorem). Indeed, by Pansu's Rademacher-type theorem [18] and its generalization to Lipschitz maps defined only on measurable sets, see [22] and [14], the Pansu-differential $d^{P} \varphi_{i}(x): \mathbb{R}^{2} \rightarrow H$ of $\varphi_{i}$ at $x$ exists for almost every $x \in K_{i}$ and is a Lie group homomorphism, equivariant with respect to the dilations. Viewed as a map between Lie algebras, $d^{P} \varphi_{i}(x): \mathbb{R}^{2} \rightarrow \mathfrak{h}$ is a Lie algebra homomorphism and therefore

$$
\left[d^{P} \varphi_{i}(x)(u), d^{P} \varphi_{i}(x)(v)\right]_{\mathfrak{h}}=d^{P} \varphi_{i}(x)\left([u, v]_{\mathbb{R}^{2}}\right)=0
$$

for all $u, v \in \mathbb{R}^{2}$. Since $\pi$ is Pansu-differentiable at $\varphi_{i}(x)$, the chain rule yields

$$
d_{x} \psi_{i}=d^{P} \pi\left(\varphi_{i}(x)\right) \circ d^{P} \varphi_{i}(x) .
$$


This together with (9) and (11) yields (10), as claimed. It now follows from (10) and (8) that for almost every $x \in K_{i}$ and all $u, v \in \mathbb{R}^{2}$

$$
P^{\prime}\left(\left[d_{x} \psi_{i}(u), d_{x} \psi_{i}(v)\right]\right)=0 .
$$

Finally, let $\left\{\xi_{1}, \ldots, \xi_{n}\right\}$ be a basis for $W_{1}$ and let $\pi^{j}: W_{1} \rightarrow \mathbb{R}$ be the corresponding coordinate functions, that is, $\pi^{j}\left(r_{1} \xi_{1}+\cdots+r_{n} \xi_{n}\right)=r_{j}$, for $j=1, \ldots, n$. Let furthermore $Q: W_{2}^{\prime} \rightarrow \mathbb{R}$ be a linear functional and set $Q^{\prime}:=Q \circ P^{\prime}$. Define functions $f_{j}: W_{1} \rightarrow \mathbb{R}$ by

$$
f_{j}(x):=Q^{\prime}\left(\left[x, \xi_{j}\right]\right)
$$

Clearly, the functions $f_{j}$ and $\pi^{j}$ are Lipschitz when $W_{1}$ is equipped with the norm $\|\cdot\|$. Furthermore, $f_{j}$ is bounded on spt $\partial \bar{S}$. We calculate

$$
\begin{aligned}
\int_{0}^{1} Q^{\prime}\left(\left[c_{1}(t), \dot{c}_{1}(t)\right]\right) d t & =\sum_{j=1}^{n} \partial \bar{S}\left(f_{j}, \pi^{j}\right) \\
& =\sum_{j=1}^{n} \bar{S}\left(1, f_{j}, \pi^{j}\right) \\
& =\sum_{i=1}^{\infty} \sum_{j=1}^{n} \int_{K_{i}} \theta_{i}(x) \operatorname{det}\left(\nabla\left(\left(f_{j}, \pi^{j}\right) \circ \psi_{i}\right)(x)\right) d x .
\end{aligned}
$$

Since, by an easy computation and (12),

$$
\sum_{j=1}^{n} \operatorname{det}\left(\nabla\left(\left(f_{j}, \pi^{j}\right) \circ \psi_{i}\right)(x)\right)=2 Q^{\prime}\left(\left[d_{x} \psi_{i}\left(e_{1}\right), d_{x} \psi_{i}\left(e_{2}\right)\right]\right)=0
$$

for almost every $x \in K_{i}$, where $e_{1}$ and $e_{2}$ are the standard basis vectors of $\mathbb{R}^{2}$, we obtain

$$
\int_{0}^{1} Q^{\prime}\left(\left[c_{1}(t), \dot{c}_{1}(t)\right]\right) d t=0 .
$$

Since $Q$ was arbitrary this shows that $P^{\prime}\left(\hat{c}_{2}(1)\right)=0$, a contradiction since $P^{\prime}\left(\hat{c}_{2}(1)\right)=$ $P^{\prime}\left(u_{0}\right) \neq 0$. This shows that there does not exist $S \in \mathbf{I}_{2}(Y)$ with $\partial S=T$. In particular, $Y$ does not admit a quadratic isoperimetric inequality for $\mathbf{I}_{1}(Y)$. Since, by Pansu's result Theorem 2.1, the unique asymptotic cone of $X:=\left(H, d_{0}\right)$ is $Y$ it follows from Theorem 3.1 that $X$ does not admit a quadratic isoperimetric inequality for $\mathbf{I}_{1}(X)$. This follows alternatively from Proposition 3.6. Consequently, by Lemma 2.3 in [25], the filling area function FA $(r)$ of $X$ cannot be bounded by $C r^{2}$ for any $C$. This completes the proof.

\section{ANOTHER LOWER BOUND}

In this section we use similar arguments as above to prove super-quadratic lower bounds for the growth of the filling area functions for another class of Carnot groups. We prove:

Theorem 5.1. Let $G$ be a Carnot group of step $k$, endowed with a left-invariant Riemannian metric. Let

$$
\mathfrak{g}=V_{1} \oplus \cdots \oplus V_{k}
$$

be a grading of the Lie algebra $\mathfrak{g}$ of $G$. If $V_{1}$ does not contain a 2-dimensional subalgebra then the filling area function $\mathrm{FA}(r)$ of $G$ grows strictly faster than quadratically:

$$
\frac{\mathrm{FA}(r)}{r^{2}} \rightarrow \infty \quad \text { as } r \rightarrow \infty .
$$


Proof. By Pansu's result, Theorem 2.1, the unique asymptotic cone of $X:=\left(G, d_{0}\right)$ is $Y:=\left(G, d_{c}\right)$, where $d_{c}$ is the associated Carnot-Carathéodory distance. Since $Y$ is geodesic and is not a metric tree, it follows for example from Proposition 3.1 in [26] that there exists $T \in \mathbf{I}_{1}(Y)$ with $\partial T=0$ and $T \neq 0$. Suppose there exists $S \in \mathbf{I}_{2}(Y)$ with $\partial S=T$. Then $\|S\|$ is concentrated on a countably $\mathcal{H}^{2}$-rectifiable subset $A \subset Y$ and is absolutely continuous with respect to $\mathcal{H}^{2}$. Since $Y$ is purely 2-unrectifiable by [15] (see also [2] for the case of the first Heisenberg group) it follows that $\mathcal{H}^{2}(A)=0$ and thus also $S=0$. As a consequence, we obtain that $T=\partial S=0$, a contradiction. It thus follows that there exists no $S$ with $\partial S=T$ and, in particular, $Y$ cannot admit a quadratic isoperimetric inequality for $\mathbf{I}_{1}(Y)$. Theorem 3.1 therefore shows that $X$ does not admit a quadratic isoperimetric inequality for $\mathbf{I}_{1}(X)$. By Lemma 2.3 in [25], the filling area function FA $(r)$ of $X$ cannot be bounded by $C r^{2}$ for any $C$.

Simple examples of Carnot groups satisfying the hypotheses of Theorem 5.1 are the first Heisenberg group and its generalizations using quaternions and octonions. It is known that the first Heisenberg group and the quaternionic Heisenberg group have cubic Dehn function, see [20] for the quaternionic case. In [20] it is also claimed that the octonionic Heisenberg group has cubic Dehn function. As was pointed out in [13] there is a sign error in the proof of the octonionic case, so that the best previously known lower bound is quadratic. From Theorem 5.1 we obtain a super-quadratic lower bound:

Corollary 5.2. The filling area function $\mathrm{FA}(r)$ of the octonionic Heisenberg group endowed with a left-invariant Riemannian metric grows strictly faster than quadratically.

For completeness, we recall the definition of the quaternionic and octonionic Heisenberg group. Denote by $\mathbb{C}$ the complex numbers, by $\mathbb{H}$ the quaternions, and by $\mathbb{O}$ the octonions. Recall that using the Cayley-Dickson construction, one obtains $\mathbb{K}_{1}:=\mathbb{C}$ from $\mathbb{K}_{0}:=\mathbb{R}$, $\mathbb{K}_{2}:=\mathbb{H}$ from $\mathbb{K}_{1}$, and $\mathbb{K}_{3}:=\mathbb{O}$ from $\mathbb{K}_{2}$ as follows: for $a \in \mathbb{K}_{0}$ define the conjugate $a^{*}$ of $a$ by $a^{*}:=a$ and the imaginary part of $a$ by $\operatorname{Im}(a):=0$. Suppose that for some $i \geq 1, \mathbb{K}_{i-1}$ has been defined together with multiplication, the conjugate $a^{*}$ of $a$, and the imaginary part $\operatorname{Im}(a)$. Set $\mathbb{K}_{i}:=\left\{(a, b): a, b \in \mathbb{K}_{i-1}\right\}$ and define multiplication on $\mathbb{K}_{i}$ by

$$
(a, b)(c, d):=\left(a c-d b^{*}, a^{*} d+c b\right) .
$$

For $(a, b) \in \mathbb{K}_{i}$, define the conjugate by

$$
(a, b)^{*}:=\left(a^{*},-b\right)
$$

and the imaginary part by

$$
\operatorname{Im}(a, b):=(\operatorname{Im}(a), b) .
$$

Now, set $\mathbb{L}_{i}:=\operatorname{Im}\left(\mathbb{K}_{i}\right)$ and define a stratified nilpotent Lie algebra $\mathfrak{g}_{i}$ over $\mathbb{R}$ of step 2 by

$$
\mathfrak{g}_{i}:=\mathbb{K}_{i} \oplus \mathbb{L}_{i},
$$

where the Lie bracket on $\mathfrak{g}_{i}$ is defined by

$$
\left[z \oplus z^{\prime}, w \oplus w^{\prime}\right]:=\operatorname{Im}\left(z w^{*}\right) \in \mathbb{L}_{i} .
$$

It is not difficult to check that for $z, w \in \mathbb{K}_{i}$ we have $\operatorname{Im}\left(z w^{*}\right)=0$ if and only if there exists $\lambda \in \mathbb{R}$ such that $w=\lambda z$. In particular, the first layer $\mathbb{K}_{i}$ of $\mathfrak{g}_{i}$ does not contain a 2-dimensional subalgebra. 


\section{REFERENCES}

[1] D. Allcock: An isoperimetric inequality for the Heisenberg groups, Geom. Funct. Anal. 8 (1998), no. 2, 219-233.

[2] L. Ambrosio, B. Kirchheim: Rectifiable sets in metric and Banach spaces, Math. Ann. 318 (2000), $527-$ 555.

[3] L. Ambrosio, B. Kirchheim: Currents in metric spaces, Acta Math. 185 (2000), no. 1, 1-80.

[4] G. Baumslag, A. Myasnikov, V. Shpilrain: Open problems in combinatorial group theory. Second edition, Contemp. Math., 296, Amer. Math. Soc., Providence, RI, 2002.

[5] G. Baumslag, C. F. Miller III, H. Short: Isoperimetric inequalities and the homology of groups, Invent. Math. 113 (1993), no. 3, 531-560.

[6] N. Brady, T. Riley, H. Short: The geometry of the word problem for finitely generated groups, Advanced Courses in Mathematics CRM Barcelona, Birkhäuser-Verlag, 2007.

[7] M. R. Bridson, A. Haefliger: Metric Spaces of Non-Positive Curvature, Grundlehren der mathematischen Wissenschaften 319, Springer, 1999.

[8] D. B. A. Epstein, J. W. Cannon, D. F. Holt, S. V. F. Levy, M. S. Paterson, W. P. Thurston: Word processing in groups, Jones and Bartlett Publishers, Boston, MA, 1992.

[9] S. M. Gersten, D. F. Holt, T. R. Riley: Isoperimetric inequalities for nilpotent groups, Geom. Funct. Anal. 13 (2003), 795-814.

[10] M. Gromov: Groups of polynomial growth and expanding maps, Inst. Hautes Etudes Sci. Publ. Math. No. 53 (1981), 53-73.

[11] M. Gromov: Filling Riemannian manifolds, J. Diff. Geom. 18 (1983), 1-147.

[12] M. Gromov: Asymptotic invariants of infinite groups, in Geometric group theory, Vol. 2 (Sussex, 1991), 1-295, London Math. Soc. Lecture Note Ser., 182, Cambridge Univ. Press, Cambridge, 1993.

[13] E. Leuzinger, Ch. Pittet: On quadratic Dehn functions, Math. Z. 248 (2004), no. 4, 725-755.

[14] V. Magnani: Differentiability and Area formula on stratified Lie groups, Houston J. Math., vol. 27 (2001), no. 2, 297-323.

[15] V. Magnani: Unrectifiability and rigidity in stratified groups, Arch. Math. 83 (2004), 568-576. by Kluwer Academic, Dordrecht, 1991.

[16] A. Yu. Ol'shanskii, M. Sapir: Quadratic isoperimetric functions of the Heisenberg groups. A combinatorial proof, Jour. of Math. Sciences, 93 (1999), no. 6, 921-927.

[17] P. Pansu: Croissance des boules et des géodesiques fermées dans les nilvariétés, Ergod. Th. \& Dynam. Sys. (1983), no. 3, 415-445.

[18] P. Pansu: Métriques de Carnot-Carathéodory et quasiisométries des espaces symmetriques des rang un, Annals of Math., 129 (1989), 1-60.

[19] P. Papasoglu: On the asymptotic cone of groups satisfying a quadratic isoperimetric inequality, J. Diff. Geom. 44 (1996), no. 4, 789-806.

[20] Ch. Pittet: Isoperimetric inequalities for homogeneous nilpotent groups, Geometric Group Theory, 159 164, Ohio State Univ. Math. Res. Inst. Publ. 3, de Gruyter, Berlin 1995.

[21] Ch. Pittet: Isoperimetric inequalities in nilpotent groups, J. London Math. Soc (2) 55 (1997), 588-600.

[22] S. K. Vodopyanov, A. D. Ukhlov: Approximately differentiable transformations and change of variables on nilpotent groups Sib. Math. Journal, 37 (1996), 62-78.

[23] S. Wenger: Isoperimetric inequalities of Euclidean type in metric spaces, Geom. Funct. Anal. 15 (2005), no. 2, 534-554.

[24] S. Wenger: Flat convergence for integral currents in metric spaces, Calc. Var. Partial Differential Equations 28 (2007), no. 2, 139-160.

[25] S. Wenger: Gromov hyperbolic spaces and the sharp isoperimetric constant Invent. Math. 171 (2008), no. $1,227-255$.

[26] S. Wenger: Characterizations of metric trees and Gromov hyperbolic spaces, Math. Res. Lett. 15 (2008), no. 5, 1017-1026.

[27] R. Young: Scaled relators and Dehn functions for nilpotent groups, preprint 2006.

Department of Mathematics, University of Illinois at Chicago, 851 S. Morgan Street, Chicago, IL 60607-7045

E-mail address: wenger@math.uic.edu 